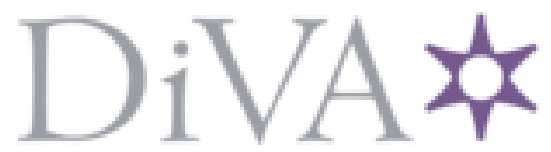

http://www.diva-portal.org

\title{
Postprint
}

This is the accepted version of a paper published in Personal and Ubiquitous Computing. This paper has been peer-reviewed but does not include the final publisher proof-corrections or journal pagination.

Citation for the original published paper (version of record):

Nazneen, N., Rozga, A., Romero, M., Findley, A J., Call, N A. et al. (2012)

Supporting Parents for In-home Capture of Problem Behaviors of Children with Developmental Disabilities.

Personal and Ubiquitous Computing, 16(2): 193-207

http://dx.doi.org/10.1007/s00779-011-0385-1

Access to the published version may require subscription.

N.B. When citing this work, cite the original published paper.

Permanent link to this version:

http://urn.kb.se/resolve?urn=urn:nbn:se:kth:diva-184689 


\section{Supporting Parents for in-Home Capture of Problem Behaviors of Children with Developmental Disabilities}

Nazneen, ${ }^{1}$ Agata Rozga, ${ }^{1}$ Mario Romero, ${ }^{1}$ Addie J. Findley, ${ }^{2}$ Nathan A. Call, ${ }^{2,3}$ Gregory D. Abowd, ${ }^{1}$ Rosa I. Arriaga ${ }^{1}$

$\{$ Nazneen, agata, mario, abowd, arriaga\}@gatech.edu

\{Nathan.Call, Addie.Findley\}@choa.org

${ }^{1}$ School of Interactive Computing, Georgia Institute of Technology, ${ }^{2}$ Marcus Autism Center, ${ }^{3}$ Emory University School of Medicine 


\section{Abstract}

Ubiquitous computing has shown promise in applications for healthcare in the home. In this paper, we focus on a study of how a particular ubicomp capability, selective archiving, can be used to support behavioral health research and practice. Selective archiving technology, which allows the capture of a window of data prior to and after an event, can enable parents of children with autism and related disabilities to record video clips of events leading up to and following an instance of problem behavior. Behavior analysts later view these video clips to perform a functional assessment. In contrast to the current practice of direct observation, a powerful method to gather data about child problem behaviors but costly in terms of human resources and liable to alter behavior in the subjects, selective archiving is cost effective and has the potential to provide rich data with minimal instructions to the natural environment. To assess the effectiveness of parent data collection through selective archiving in the home, we developed a research tool, CRAFT (Continuous Recording And Flagging Technology) and conducted a study by installing CRAFT in eight households of children with developmental disabilities and severe behavior concerns. The results of this study show the promise and remaining challenges for this technology. We have also shown that careful attention to the design of a ubicomp system for use by other domain specialists or non-technical users is key to moving ubicomp research forward.

\section{Author Keywords}

Selective archiving, problem behavior, direct observation, behavior assessment, recording and flagging.

\section{Introduction and Background}

Epidemiological studies by Emerson and McDougal \& Hiralall suggest that $13 \%$ to $30 \%$ of young children engage in problem behaviors that require intervention [1-3]. Problem behaviors are common in children with developmental disabilities, including autism, which alarmingly affects $1 \%$ of children in the United States [4-6]. Problem behaviors, such as physical aggression, selfinjury, stereotypy, disruption, and tantrums, have a severe effect on educational and social development and could become a regular occurrence in a child's daily life without early intervention [7-9]. Ignoring this pervasive problem in the hopes that it will improve with age may only worsen the situation $[10,11]$. Thus, many early intervention programs include procedures for behavioral assessment and intervention for problem behaviors [12, 13]. 


\section{Direct Observation of Problem Behavior for Assessment}

Behavioral intervention requires the understanding of topography (characteristics) as well as the function (the antecedent variables that evoke and/or the consequences that maintain) of problem behavior [14-21]. Direct observation of the subject is considered the gold standard in effective assessment of problem behaviors [22, 23]. However, direct observation introduces a number of challenges. Based on our literature review and discussions with behavior analysts, we have identified some of the major challenges as context, validity, sparsity, safety, and resourcing [2426].

- Context: Stimuli that evoke the problem behavior (i.e., antecedents) as well as consequences (events that follow the problem behavior) that maintain it in the natural environment may not exist in an artificial setting such as a clinic.

- Validity: The presence of an unfamiliar observer in a home, school, or clinic setting may cause children to alter their behavior due to their awareness of being observed. Such reactivity effects pose a threat to the validity of collected data.

- Sparsity: If the problem behavior occurs infrequently, the chances of collecting sufficient samples during limited observation periods are diminished.

- Safety: Direct observation of aggressive behaviors presents a safety risk to the observer.

- Resourcing: The number of children in need of intake assessments for severe behaviors far outnumbers the staff available to provide the needed services. At the Marcus Autism Center, a state-of-the-art local severe behavior clinic, it is common for parents to be put on a waiting list for several months before their child receives services.

\section{Video Observation of Problem Behavior for Assessment}

Video observation represents one avenue for tackling the challenges of direct observation. Typical video recording methods use either continuous recording or discrete recording. In continuous recording, the video is recorded uninterrupted and the goal is to capture instances of the target behavior by collecting video across several hours or days. Unfortunately, this approach generates vast volumes of data that are prohibitively labor intensive to analyze manually, especially if the occurrence of the target behavior is sparse. The second approach is to record discrete instances of problem behaviors as they occur. The setback with traditional discrete recording is that the temporal context of the target behavior is inadequately captured. When the observer triggers the 
system, the annotated recording typically misses the moments before the trigger that are crucial to determining the cause of a target behavior. Selective archiving is an advanced discrete recording method that continuously captures a temporal buffer but only stores it when the user explicitly triggers the system to record $[27,28]$. When the observer triggers the system, the moments prior to the trigger are also captured, thus preserving the temporal context. We argue that video recording through selective archiving is an alternative to address many of the challenges of direct observation and traditional continuous and discrete video recording.

\section{Parents as Collectors of Evidence of Problem Behaviors}

We posit that giving parents access to video recording tools like selective archiving will enable them to collect video samples of child problem behaviors for later analysis by behavior experts. We further posit that there are incentives for parents and professionals to adopt such a technology approach if it demonstrates to be more effective than current practices. For instance, the biggest motivation for the behavioral clinic to participate in the evaluation of the technology was the possibility that ultimately they could ship a recording system for parents to collect samples of a child's problem behaviors, in lieu of bringing the child in for an observation at the clinic or sending a trained observer to the child's home. This would be a cost effective solution, streamline the intake process, and potentially help the clinic reach a larger population, including those located far from the clinic (e.g., rural areas). Moreover, because the assessment and treatment course would now be based on evidence of problem behaviors captured in a natural setting, the clinic would be getting a more ecologically valid picture of the antecedents and consequences of the child's problem behavior. For parents, the potential for the system to reduce the length of time spent on the waiting list for an intake assessment means that they would be able to get early and timely intervention for their child. However, before such technology is widely adopted, it is necessary to examine the kind of data that parents are able to collect and determine whether this data is sufficient for a behavior analyst to make their assessment.

\section{Contributions of This Paper}

In collaboration with the severe behavior clinic at the Marcus Autism Center in Atlanta, we designed an exploratory study to begin to understand the utility of an in-home, video-based, problem behavior capture system. There are two main contributions of this paper, one relating to 
the specific application domain of behavioral health, and one relating to the adoption of ubicomp technologies in the real world.

- From the perspective of the domain of behavioral health, we present a novel exploratory study of eight households with children with autism and related disabilities. We describe the data collected by parents when asked to flag "problem behaviors" of their children. We also compare the parent's annotations against a standard coding scheme used by professionals (i.e., behavior analysts) to ascertain where the overlap and differences lie between what is considered "problem behavior" by parents and professionals. We present lessons learned concerning the potential role that technology can play in improving data collection in the natural environment. We explore whether the results of this initial study can be improved upon, both by better training as well as by increased use of technology.

- Of general interest to the ubicomp research community, we present a case study of a system in the home that is to be deployed and operated exclusively by individuals who do not have a technical background, and discuss the constraints that this imposed on the design of the system.

\section{Related Work}

There is growing interest in research towards the design of technologies to capture data in the natural environment for behavior assessment and intervention of children with developmental disabilities. Here, we will discuss some relevant work in this area, namely video capture of child behavior in natural settings for subsequent analysis.

The Walden Monitor (WM) was an experimental system designed to collect observation data of children with autism in a specific inclusive educational setting [29]. It included a wearable camera and a tablet PC as an alternative to paper-based data collection methods. CareLog was also an effort in the same direction where the goal was to facilitate record keeping of children with autism by teachers, and introduced the idea of selective archiving as an interesting subset of automated capture in ubicomp [27]. Hayes et al. define selective archiving as a capture and access model that constantly buffers data but permanently stores it only when explicitly triggered by some external event, either by a human in the scene or some automatically determined criteria. Selective archiving supports the potential for deeper reflection on the context of a recorded event, in this case behavioral problems. A pre- and post-trigger window of data is stored, effectively supporting 
retroactive recording of what occurs in a live setting around the triggered event. This approach has several benefits. First, when triggering occurs by manual input, it puts the user in complete control of when the data is stored, simultaneously addressing privacy and storage management concerns that arise from continuous data capture, especially with video recording. Second, with the human in the loop, irrelevant data is implicitly filtered, saving resources both in the present (disk space) and in the future (time spent analyzing the data).

A commercial implementation of selective archiving, Behavior Capture ${ }^{\mathrm{TM}}$ by Behavior Imaging Solutions [30] is now making that service available for use in a variety of clinical and educational settings with trained personnel. The opportunity for using Behavior Capture ${ }^{\mathrm{TM}}$ in a home setting is what inspired our current work. The central unanswered question for Behavior Capture ${ }^{\mathrm{TM}}$ is whether parents can effectively flag target behaviors. This is the main research question of this paper. Another limitation of Behavior Capture ${ }^{\mathrm{TM}}$ is that there is only one camera, which limits the data collection area. The orginal CareLog study showed that multiple, overlapping camera views was beneficial to understanding the complexity of a child's behavior in the classroom. For home use, we hypothesize that a selective archiving system should also support multiple cameras. This should preferably be wireless to avoid wire clutter. Parents should also have the freedom to trigger the recording from any part of the home.

In the CareLog study, significant effort went into training teachers to identify and define the behaviors that were the target of subsequent data collection. Since this is the first study in a home environment, we opted against training parents for an exploratory design. Instead, we simply asked parents to flag problematic behaviors. In this manner, we were able to get at their intuitive sense of what constitutes a problem behavior. In addition, the CareLog system was designed for capturing behavior data in a confined classroom. The home is a more dynamic space where the child has the freedom to move from room to room. This poses technical challenges to ensure camera coverage of the child's movements. Moreover, in a classroom setting, the teacher is dedicated to watching over the children whereas parents may be busy with other household routines.

In this paper, we describe the use of selective archiving technology in the home. The goal of this study is to explore the role that selective archiving can play between parents of children with problem behaviors and the professionals that are ultimately entrusted with designing a treatment for these behaviors. We investigate the data parents collect with the goal of understanding what 
behaviors are deemed "problematic" by parents and to ascertain which of the behaviors the parents flagged meet the clinical criteria for problem behaviors by professionals.

With this background motivation for the study, we now describe the experimental system and research protocol developed to explore parent data collection in the home.

\section{CRAFT Research System}

To carry out this exploratory study, we developed a capture and access system, CRAFT (Continuous Recording And Flagging Technology). The purpose of CRAFT is two-fold:

1. To simulate user experience of selective archiving. CRAFT does not explicitly implement selective archiving. Parents are asked to flag incidents of problem behavior but, unlike select archiving, these flags do not trigger the recording of video.

2. To continuously record in order to facilitate comparisons between parent annotations and coding conducted by a behavior analyst using specific coding criteria used by the severe behavior clinic. Continuous recording allows the behavior analyst to review and determine all instances of problem behavior that meet the clinic's criteria for problem behavior. The behavior analyst coding is then compared to parent flags to note agreements and disagreements.

A functional requirement for CRAFT, therefore, is to support robust and continuous capture from multiple synchronized cameras as well as time-stamped events triggered by the human via a small wireless device. Parents used the wireless device to flag the onset of their child's problem behavior. A second functional requirement of CRAFT is an access interface that allows behavior analysts to review the recorded video and compare their coding of the child's problem behaviors against instances of these behaviors flagged by parents. In the following section, we separately describe these two features.

\section{CRAFT Capture System}

The Capture System is designed so that it can be deployed in a typical home. The physical installation (see Figure 1) supports up to four network cameras to capture video, a wireless remote to facilitate parent annotations, a receiver for the wireless remote, and a portable computer (UMPC) with wireless network capability that hosts a fault-tolerant video capturing system. We limited the number of cameras to four to ensure that the video quality did not suffer due to the increased load on the portable PC both in terms of network bandwidth and processing power. 
The Capture System supports up to four Axis 207W wireless network cameras synchronized using a NTP server. The system records video continuously until the user stops it or the recording reaches a pre-defined auto-stop time limit. The cameras stream video at 30 frames per second (fps) with VGA resolution (640 by 480 pixels). Three cameras connect wirelessly while the fourth is wired. The wired camera ensures high quality video from at least one source. We used wide angled lenses on the cameras to have better coverage with fewer cameras.

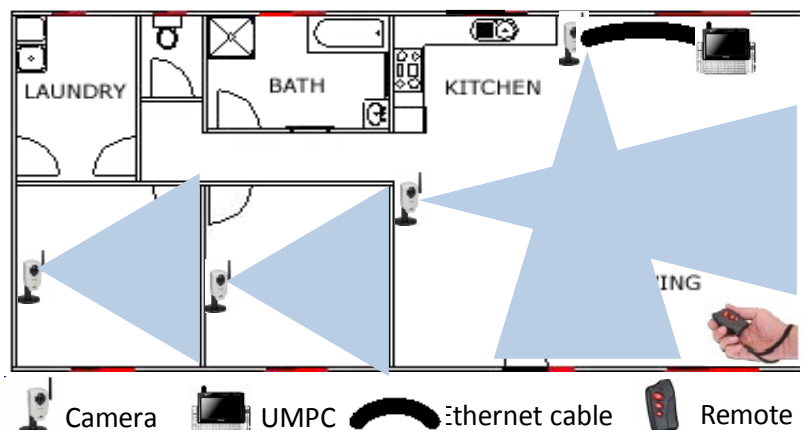

Fig. 1 A typical CRAFT home installation. The camera closest to the portable PC uses a wired Ethernet connection. All other cameras communicate via a local wireless network directly to the UMPC

Figure 2 shows a screenshot of the user interface seen in the home during operation. The colored circles next to the cameras represent the status of each camera. Green indicates that the camera is online and the video is being recorded, whereas red indicates that the camera is offline. A large, central button starts and stops the video recording. The system anonymizes the data by using a unique code for each participant, and stores it in a secured repository to which only authorized study personnel have access.

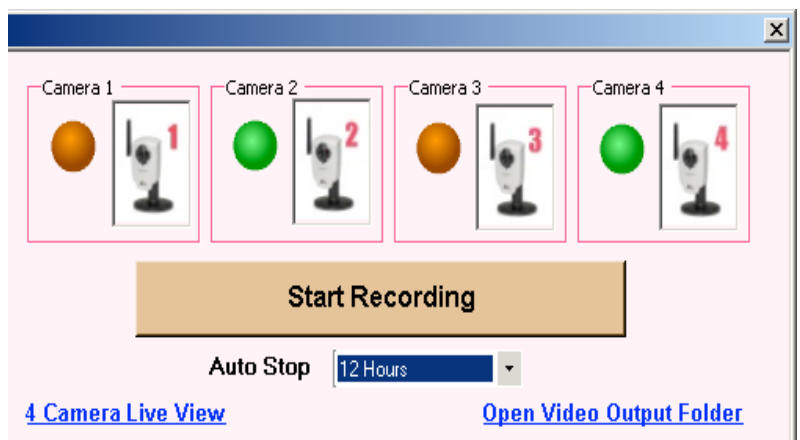

Fig. 2 The CRAFT Capture System Interface

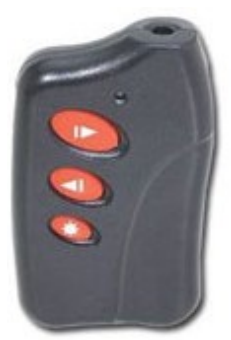

Fig. 3 Honeywell RF Remote

To flag the occurrence of problem behaviors, parents use a lightweight Honeywell RF remote control (see Figure 3) with a range of $150 \mathrm{ft}$., sufficient to cover an average-sized home. This remote is an off-the-shelf product designed for remote input to a laptop. It has three buttons, a "forward" event, a "backward" event, and a button to engage a laser pointer. The laser pointer 
button was covered to prevent its use, while the other buttons served the same purpose, to flag the occurrence of a problem behavior. A host process on the portable PC receives button-click events, tags them with a time stamp, and stores them as parent annotations. The output files from each recording session include the video from each camera and a log of the parent-initiated button clicks from the remote. The system also keeps track of each camera's disconnection time to support synchronized playback in the access system, described next.

\section{CRAFT Access System}

We created a basic interface (Figure 4) for the behavior analyst to view the synchronized video feeds from multiple cameras using Microsoft's Media Player, the AviSynth frame server scripting program [31], and the logs generated from the CRAFT Capture System.

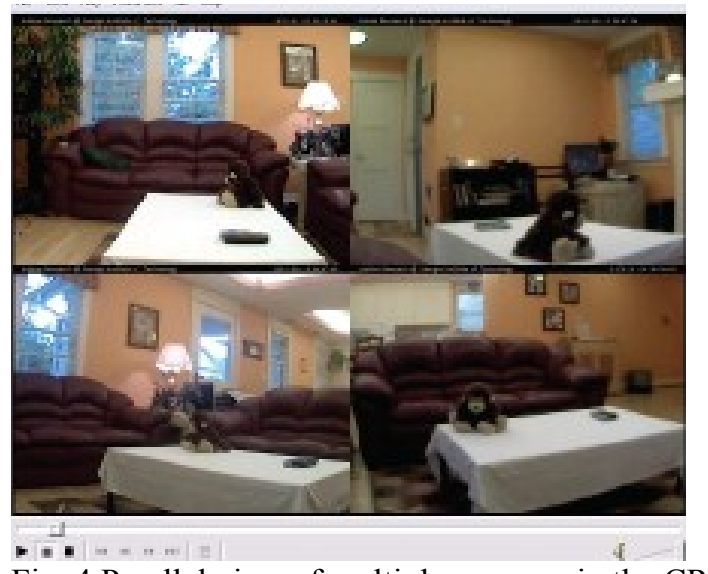

Fig. 4 Parallel view of multiple cameras in the CRAFT Access System

\section{Design and Deployment Considerations}

Through a series of pilot deployments in homes of neurotypical children and children with autism, we identified a number of additional issues that needed to be addressed to make CRAFT a robust, autonomous, and unobtrusive recording system.

\section{Design for Minimal User Effort}

There are three user populations for CRAFT: the research assistants (not ubicomp researchers) that will deploy the system, the parents as annotators, and the behavior analysts as coders that analyze the collected data. A key design feature is ease of deployment since the research assistants do not have a technical background. Thus, the placement of cameras and system setup needed to be straightforward. Likewise, ease of use was important for the parent, such that the interface would not interrupt their daily routine (for example, by requiring special attention). We needed to ensure that when a child is exhibiting a problem behavior, relatively little time is lost annotating the 
event, as the parent's time is better spent attending to their child. Moreover, any response that requires the parent to allocate significant time away from their child is likely to decrease their willingness to use the system. Similarly, it is crucial that the startup process proceeds smoothly since the users are not computer technicians and may not be able to easily debug installation failures. Furthermore, it is paramount that once the recording process is underway, the system operates without any further interaction on the part of the user, until recording is finished.

We adopted the following strategies to minimize the need for user input and effort:

\section{Single Click Approach}

For parents, in order to keep the mental and physical demand low, the interaction with the system is limited to a single button click on the remote control. Deployment involved positioning and powering up cameras and then pressing the 'Start Recording' button in the interface. In this study, the clinic's staff carried out the deployment, but eventually we envision that the behavior clinic will ship the system to parents who will mount the cameras themselves and start the recording.

\section{Location independence}

The long-range remote ensures that parents can use it confidently from any place within the home.

\section{Visual Interface}

Our user interface design uses visual color-coding to reflect each camera's status. This makes it intuitive for the users to determine when the cameras are offline (red) or online (green).

\section{Contingency Management}

There are many reasons for a camera to become disconnected from the recording service (e.g., being unplugged, wireless connection issues). In order to avoid having the user troubleshoot connection problems, a watchdog service that monitors and restarts connections to the cameras upon discovery was built into the CRAFT system.

\section{Single Synchronized Data View}

A single synchronized view from multiple cameras is provided to facilitate ground-truth coding by the behavior analyst.

\section{Addressing Privacy Concerns Through User Control and Transparency}

Privacy is a serious concern when it comes to video recording in homes, perhaps more so than in educational or therapy settings, and can be addressed by providing the participants who are being 
recorded with complete control over the system and data collection [32]. We took the following steps to address privacy concerns of the participants:

\section{Control Over Capture}

We include the option for parents to terminate the recording at any time by simultaneously clicking two buttons on the remote, which stops the recording process and deletes all collected data. To avoid accidental deletion, the PC flashes a warning message requiring further confirmation from the parents. Additionally, the CRAFT system provides the flexibility to use any number of cameras (up to a maximum of four) depending on the user's privacy needs.

\section{Transparent Capture}

Participants being recorded also need to know what is being recorded and where. For this purpose the system provides a live view option, where the video feed from the multiple cameras is shown in a single window. It is also important to make the field of view of the cameras explicit in the recording environment. We placed tape on the ground marking the boundaries of the field of view for each camera. The physical marking of the boundary of the fields of view was intended to make clear the area of capture, allowing participants to simply exit or take the child out of the area when they did not want to be recorded. The physical marking was also intended to help the parents maintain the action in the field of view when they were seeking to collect samples of a target behavior.

\section{Data Protection}

Privacy protection is central to any ubiquitous computing system deployed in such a sensitive environment. In addition to password protection, the privacy of participants is safeguarded by configuring the cameras such that only a limited number of clients can access the transmitted video on the local wireless network, which is secured by a WEP (Wired Equivalent Privacy) key. The overall system does not connect to any external network.

\section{Final Say Over Data Usage}

When the system was deployed, parents were taught how to delete data they had collected. One method that has already been mentioned is simultaneously clicking both remote buttons during data capture. The other method is by accessing the folder where the video and output files are stored using the "Open Video Output Folder" link on the interface (see Figure 2), and deleting all the files in that folder. After the deployment, when the clinic's staff came to pick up the system, parents were once again reminded that they could choose to delete all the data they had recorded. 


\section{Study Design}

\section{Participant Recruitment}

Through our collaboration with the severe behavior clinic at the Marcus Autism center, we recruited eight families, each with a child diagnosed with a developmental disability. The children were all male and ranged in age from $4-19$ years $($ Mean $=9.8$ years, Std. Dev. $=4.7)$. Two of the families were participating in a clinic-based intervention program and the remaining families were participating in a home-based treatment program, with both programs administered by the severe behavior clinic. The clinic's staff recruited the participant families during one of the families' regularly scheduled visits to the clinic. The recruitment was verbal and we did not provide monetary incentives or any other form of compensation.

\begin{tabular}{|c|c|c|l|l|c|}
\hline Child & Age & Gender & Ethnicity & Diagnosis & $\begin{array}{l}\text { Intervention } \\
\text { Program }\end{array}$ \\
\hline C1 & 10 & M & Caucasian & Autism & Home \\
C2 & 9 & M & Caucasian & $\begin{array}{l}\text { Disturbance of Conduct-Not } \\
\text { Otherwise Specified }\end{array}$ & Clinic \\
C3 & 6 & M & Unspecified & Autism & Home \\
C4 & 6 & M & Caucasian & Autism & Home \\
C5 & 19 & M & Egyptian & Pervasive Developmental Delays & Clinic \\
C6 & 4 & M & Caucasian & Autism & Home \\
C7 & 9 & M & Unspecified & Asperger & Home \\
C8 & 15 & M & Caucasian & Autism & Home \\
\hline
\end{tabular}

Table 1. Participant demographics

\section{Deployment Protocol}

Staff from the clinic visited participating homes to set up the CRAFT Capture System. Parents were asked to choose the number of cameras they felt comfortable with and to determine the locations in their home where they considered problem behaviors most likely to occur. It was important to let parents decide on the number of cameras and not enforce the use of all four cameras since this helped alleviate their privacy concerns and may have lowered the chances of them opting out of the study at the time of deployment. Once the cameras were set up, the researcher instructed the parents to click a button on the remote every time they observed the onset of a behavior that they considered a problem behavior. They were told that if the child engaged in the same behavior repeatedly over a short span of time, they only needed to click once.

\section{Data Coding}

After a one-day deployment the clinic's staff went back to collect the system. Owing to the large volume of data collected (average of 12 hours across the eight families), the task of coding the 
videos was divided among ten behavior analysts from the severe behavior clinic. The behavior analysts viewed the videos captured via continuous recording and applied a coding scheme based on standard definitions of problem behaviors used by the clinic in their daily practice (see Table 2). To increase confidence in the validity of each analyst's coding, a second behavior analyst coded an average $20 \%$ of the data that was randomly selected across all participants. Inter-observer agreement was greater than $90 \%$ across all participants. During deployment, for ease of use, parents were asked to click once for similar instances of a problem behavior that occurred within 15 minutes. In line with the instructions given to the parents, while coding the data, instances of similar problem behavior that occurred within a 15-minute span were counted as a single instance of that behavior. Upon completion of the coding, the behavior analysts compared their codes to the behaviors flagged by the parents.

\begin{tabular}{|l|l|}
\hline Category & Operational Definition \\
\hline Aggression & $\begin{array}{l}\text { Any instance of hitting another individual with an open or closed fist from a } \\
\text { distance of } 6 \text { inches or greater, kicking others from a distance of } 6 \text { inches or } \\
\text { greater, scratching, pinching, pulling hair, grabbing, biting, shouting. } \\
\text { Any instance of throwing objects (other than during appropriate toy play), } \\
\text { ripping, crumbling and tearing objects, banging walls and/or any hard surface } \\
\text { with an open or close fist from a distance of } 6 \text { inches or greater. }\end{array}$ \\
Self-Injury & $\begin{array}{l}\text { Any instance where the head comes in contact with any object in a forceful } \\
\text { manner from a distance of three inches or greater, or any time the child slaps } \\
\text { their face or head from a distance of three inches or greater, bites himself, digs } \\
\text { their chin into another body part, or pokes his or her own eyes. }\end{array}$ \\
Flop & $\begin{array}{l}\text { Any instance in which the child drops to his or her knees or lower without the } \\
\text { consent of the caregiver. } \\
\text { Any instance of moving or attempting to move } 3 \text { feet or more from the caregiver, } \\
\text { any instance in which the child grasps or turns the door handle in the attempt to } \\
\text { exit the room. }\end{array}$ \\
\hline
\end{tabular}

Table 2. Standard operational definitions of problem behaviors used by behavior analysts to code the home deployment data

\section{Study Results}

\section{System Deployment}

Clinic's staff was trained to deploy the system in two pilot homes and in one home in this study. After that initial training they were able to deploy and collect the CRAFT system in the other seven homes without any problems (i.e., they did not have to call for technical support).

Of the eight parents in the study, only one reported a complication with the system. They had to hand off the remote to the other parent because they were in the middle of something and could not click it themselves. 


\section{Data Description}

In this section we present the data collected by parents as well as that coded by the behavior analysts. The specific categories of problem behavior identified by the behavior analysts while coding the continuously recorded video obtained from each family are listed in Table 3 . The second column lists the problem behavior category from Table 2 that each participant engaged in during the observation period. The third column lists the exact topography of each behavior under that category.

\begin{tabular}{|c|c|c|}
\hline Family & Problem Behavior(s) Category & Exact behaviors \\
\hline F1 & Flop \& Elope & Flopped to the ground, ran away (eloped) \\
\hline F2 & Disruption \& Elope & $\begin{array}{l}\text { Throws objects such as toys, shirts, blankets. } \\
\text { spills water on floor, elope }\end{array}$ \\
\hline F3 & Aggression & Kicking people \\
\hline F4 & Aggression \& Disruption & $\begin{array}{l}\text { Throws objects such as doll, cat, dustpan. Hit } \\
\text { brother with dustpan. Flipping cat }\end{array}$ \\
\hline F5 & Self-Injury \& Disruption & Hit his chest and throws objects \\
\hline F6 & Disruption, Flopping and Aggression & $\begin{array}{l}\text { Tearing objects, flop, Hitting, kicking, slaps, } \\
\text { hits brother }\end{array}$ \\
\hline F7 & Self-Injury and Aggression & Bites, shouting, kicking people \\
\hline F8 & None Observed & None observed \\
\hline
\end{tabular}

Table 3. Child's problem behavior(s) based on coding scheme established by the behavior analysts

A summary of the data showing the number of parent clicks and the coded episodes of problem behavior by the behavior analysts is presented in Table 4. Across the eight participant families, parents flagged 46 episodes of problem behavior whereas behavior analysts identified 33 episodes.

To perform a more in-depth analysis and determine the type of data collected by parents, we further classified this raw data into five categories. The definitions of these categories are provided in Table 5.

\begin{tabular}{|c|c|c|}
\hline Family & Parent Flags (Clicks) & Behavior Analyst Coding \\
\hline F1 & 8 & 1 \\
F2 & 11 & 9 \\
F3 & 0 & 1 \\
F4 & 0 & 8 \\
F5 & 14 & 7 \\
F6 & 11 & 6 \\
F7 & 2 & 1 \\
F8 & 0 & 0 \\
Totals & $\mathbf{4 6}$ & $\mathbf{3 3}$ \\
\hline
\end{tabular}

Table 4. Total number of target problem behaviors per participant family identified through parent annotations and behavior analyst coding 


\begin{tabular}{|l|l|}
\hline Variables & Description \\
\hline Match & $\begin{array}{l}\text { Parents and Behavior Analysts concur on the identification of problem } \\
\text { behavior. } \\
\text { Criteria: Parent clicked the button within } 15 \text { minutes of a behavior that } \\
\text { Parent identifies a problem behavior but behavior analyst does not concur } \\
\text { with the parent's assessment. } \\
\text { Criteria: Child was within the camera's view but no target event was } \\
\text { coded by the behavior analyst within } 15 \text { minutes of the parent click. } \\
\text { Behavior analyst codes problem behavior but parent does not flag that } \\
\text { Mehavior } \\
\text { Criteria: Parent and child were within the camera's view. Target event } \\
\text { was coded by the behavior analyst but there was no parent click. } \\
\text { Behavior analyst codes a problem behavior but the child is alone within } \\
\text { the view of the camera. } \\
\text { Criteria: Child was within the camera's view and engaged in a problem } \\
\text { behavior, but the parent was out of the camera's view and therefore did } \\
\text { not click. } \\
\text { Parent click detected but the child is out of the camera's view. } \\
\text { Criteria: A parent click was detected but no problem behavior was } \\
\text { observed in the 15 minutes prior to the parent's click and the child was } \\
\text { not within the camera's view for more than 15 consecutive seconds. }\end{array}$ \\
Child Out of \\
Scene
\end{tabular}

Table 5. Criteria used in comparing parent annotations against the behavior analysts' coding

Based on the categories from Table 5, Table 6 provides a detailed split of the data shown in Table 4. The total number of parent clicks in the study can be obtained by summing the Match, Flag Mismatch and Out of Scene columns. To obtain the total number of behaviors coded by the analysts, sum the Match, Coding Mismatch and Alone columns.

Across the eight families, the analysts identified 20 episodes of problem behavior that met the criteria listed in Table 5. These were instances in which the parent was present and thus should have flagged the behavior via a remote click. Out of these 20, parents flagged the behavior (by clicking the remote) for $11(55 \%)$ of the episodes (Match) and did not flag 9 (45\%) instances (Coding Mismatch). Moreover, parents identified an additional 19 events that did not fit the standard operational definitions of problem behaviors used by behavior analysts (Flag Mismatch).

\begin{tabular}{|c|c|c|c|c|c|}
\hline Family & *\#Match & $\begin{array}{c}\text { *Flag } \\
\text { Mismatch }\end{array}$ & $\begin{array}{c}\text { \#Coding } \\
\text { Mismatch }\end{array}$ & \#Alone & *Out of Scene \\
\hline F1 & 1 & 3 & 0 & 0 & 4 \\
\hline F2 & 3 & 7 & 2 & 4 & 1 \\
\hline F3 & 0 & 0 & 0 & 1 & 0 \\
\hline F4 & 0 & 0 & 1 & 7 & 0 \\
\hline F5 & 2 & 7 & 4 & 1 & 5 \\
\hline F6 & 4 & 1 & 2 & 0 & 6 \\
\hline F7 & 1 & 1 & 0 & 0 & 0 \\
\hline F8 & 0 & 0 & 0 & 0 & 0 \\
\hline Total & 11 & 19 & 9 & 13 & 16 \\
\hline
\end{tabular}

Table 6. Comparison of parent-identified problem behaviors against the behavior analyst's coding scheme. * Sum of these indicates total number of parent clicks \# Sum of these indicates total number of behaviors coded by analysts 
Thirty-nine percent of the problem behaviors coded by the behavior analyst occurred while the child was Alone. In addition, $34 \%$ of all parent clicks fell into the Out of Scene category and thus could not be coded by the behavior analyst. The unique issues associated with these two categories will be discussed in the next section.

\section{Discussion}

Selective archiving can be used to collect observations in the natural environment and will not only address limitations of direct observation but will also allow parents to play a role in the assessment of problem behaviors and intervention for their child. However, this study shows that parents and behavior analysts diverge in what they consider problem behavior. In this study, parents were asked to annotate their child's problem behavior via a click. Our goal was to describe the data that parents collected (without training) and then to compare it to the coding scheme that trained professionals use to define problem behavior. In this section we: 1) describe what behaviors are deemed "problematic" by parents; 2) ascertain which of the behaviors the parents flagged meet the operational criteria for problem behavior utilized by the behavior analysts and describe instances that lead to discrepancies between parents and behavior analysts; 3) reflect on whether the data collected by parents (without training) can be used to assess the functional aspects of the severe behavior; and 4) reflect on how selective archiving addresses some of the challenges of direct observation of problem behavior that we identified in the introduction.

\section{Behaviors Annotated by Parents}

In this study we set out to describe the behaviors that parents considered to be problem behaviors. Our goal was to use their intuitive understanding of these behaviors rather than train them to flag a particular behavior. We took this approach because this is the first study of its type and we wanted to avoid inserting procedures into the protocol that would make it more expensive and cumbersome, such as training the parents on specific types of problem behaviors. We also wanted to observe what types of behaviors would cause parents sufficient concern to bring their child to a severe behavior treatment clinic in the first place. We found that there were five types of behaviors that were deemed to be problematic by parents: flopping, eloping, disruptions, aggression and selfinjury (see table 2 for the operational definitions of these behaviors). Aggression and disruption were the most commonly reported problem behaviors, with four of the seven parents flagging 
instances of these behaviors. We also found that six of the seven parents reported more than one problem behaviors.

\section{Comparing Parent's Intuitive Annotations to Behavior Analysts' Criteria Based Assessments}

Another goal of this study was to ascertain which of the behaviors the parents flagged meet the clinical criteria for problem behavior by professionals. A comparison of parent annotated data and data coded by the behavior analysts resulted in five categories of data: Match, Flag Mismatch, Coding Mismatch, Alone and Out of Scene. Matches are episodes of problem behavior that were identified by both the parents and the behavior analysts. In the present study, parents and behavior analysts agreed 55\% of the time. Flag Mismatch and Coding Mismatch represent instances where parents and analysts did not agree on whether a behavior was problematic, respectively. Each of these instances present distinct challenges to how the behavior analyst can use the footage provided by the parent. If we consider that ultimately a system like CRAFT would be deployed in households with only the selective archiving feature, without the additional continuous recording mode, the consequence of a Flag Mismatch will be that the behavior analyst will spend some time reviewing footage that ultimately will not meet the clinic's criteria for severe problem behavior. However this may also present an opportunity for the analyst to understand what the parent views as problematic, and may facilitate dialogue between the two parties so that parents better understand why a professional may choose to intervene for one behavior and not the other. In contrast, the consequence of a Coding Mismatch is more serious, as important clinical information (i.e., an occurrence of problem behavior and the associated environmental events) is lost because the parent does not flag the behavior in the first place. A domain-specific (as opposed to technical) research question that stems from this finding is whether interviews with parents and/or parent training prior to deployment would decrease instances of Coding Mismatch. Also, are parents more easily trained to flag some behaviors than others? Another related question is whether training parents is a cost-effective option. It may be that simply increasing the length of time the system is deployed in a family's home leads to an increased number of instances that behavior analysts can use to make their assessment.

We identified two additional challenges to the use of selective archiving to collect evidence of severe problem behaviors in the home. The first of these, Alone, relates to instances when the parents are not present when the child engages in a target behavior. Since parents cannot be 
expected to be near their child at all times, a selective archiving system which only stores video when explicitly triggered by parents will likely miss such events. We propose that a possible solution to this issue is to complement explicit parent triggers with an implicit triggering mechanism via the automatic detection of problem behaviors. To give one example, the system could be designed to detect shouting, and automatically trigger video recording when this target behavior occurs. An additional technical consideration would be that the video should clearly indicate that this was automatically flagged since behavior analysts would need this information to understand the context in which the behavior occurred. Further proposals for implicit triggering are discussed below in the "What Lies Ahead" section.

A second challenge, Out Of Scene, is presented by circumstances where parents have triggered the recording system but the child is not in the camera's view, and thus the behavior analyst reviewing the flagged annotations cannot determine whether he agrees or disagrees with the parent. Improving the coverage of the cameras can minimize this issue but cannot completely eliminate it as we cannot reasonably expect to place cameras everywhere in the house. For example if the problem behavior occurs while the child is hiding under the table then it becomes extremely difficult to capture video of this behavior with complete context. Another possibility is for the system to provide feedback to inform the parent that no child behavior was captured, assuming we could reliably determine that the child was not within camera field of view.

\section{Can Data Collected by Parents be Utilized by Professionals to Make Clinical Assessments?}

While there are a number of technological solutions that we envision could reduce the impact of the challenges noted above, it is important to step back and consider one goal of the data collection described in this paper, namely its use by a behavior analyst to assess the functional aspect of the severe behavior. The clinicians at the severe behavior clinic need to see the child engaging in target problem behaviors so that they can assess the nature and severity of the problem, and determine the right course of treatment. In our discussions with the clinicians it became clear that in fact it might not be necessary for them to observe every instance of problem behaviors. Rather, they need sufficient samples of each problem behavior in question to be able to reach the same conclusions about the antecedents and consequences of the behavior as they would based on a brief observation at the clinic or in the child's home, as is standard practice. The question of how many examples are sufficient is an empirical one. Analysis of the Matches in the current data 
revealed that five parents flagged at least one instance of each problem behavior identified by the behavior analyst. In the behavior analysts' opinion, if the CRAFT system was deployed for a longer period of time, for example one week rather than just a day, it would increase the likelihood that a sufficient number of examples of problem behavior were collected across all families.

\section{Does Selective Archiving Ameliorate the Challenges of Direct Observation?}

Direct observation is considered the gold standard for the effective assessment of problem behaviors [22, 23]. However, it introduces a number of challenges we identified in our introduction, including context, validity, sparsity, safety, and resourcing [24-26]. Below we discuss what our study suggests are the implications of using selective archiving instead of direct observations.

- Context: we proposed that selective archiving might facilitate the observation of naturally occurring antecedents and consequences of problem behaviors in the course of the child's day-to-day activities. Our study suggests that video footage collected via selective archiving can be coded by the behavior analyst using existing criteria for severe behavior problems.

- Validity: we noted that the presence of an unfamiliar observer, even in a home, school or clinic setting, may cause children to alter their behavior due to their awareness of being observed. Such reactivity effects pose a threat to the validity of any collected data. In the current study, only one child (a 19-year-old male, the oldest in our sample) demonstrated an awareness of the cameras. Specifically, he initially unplugged all of the cameras, requiring the clinic staff conducting the deployment to take extra care in hiding them. Thus, largely we did not see evidence that the children were aware of the cameras, suggesting that selective archiving may alleviate the issue of reactivity in this population. However, we note that selective archiving may lead to reactivity in the parent. Parents know that their decisions about the child's behavior are being scrutinized and this may lead them to behave differently, which may in turn affect the child's behavior. Future research could address this concern by interviewing the parent regarding how they felt about having the cameras on. One can even imagine a controlled experiment where "awareness of being recorded" is manipulated (via a signal on the camera) and researchers measure change in behavior of the parent or the child.

- Sparsity: we noted that for problem behaviors that occur infrequently, the chances of collecting sufficient samples during limited observation periods are slim. Selective archiving 
may ameliorate this problem because the deployment can be of a longer duration than what is usually scheduled for a direct observation. In our study the average deployment was 12 hours compared to the two hours that a live observer would spend in the home. However, this study also suggests that 12 hours may not be sufficient time to capture some instances of severe behavior using selective archiving. For example, three of the eight families indicated no instances of the severe behavior and a fourth family indicated only two instances. This suggests that the selective archiving system should be deployed for longer time periods.

- Safety: we noted that direct observation of aggressive behaviors presents a safety risk to the observer. We propose that selective archiving can alleviate the safety concern inherent in direct observations of aggressive behaviors by replacing the observer with cameras and parent annotations via a remote control.

- Resourcing: The number of children needing intake assessments and services for severe behaviors far outnumbers the staff available to provide these services. We propose selective archiving can address this challenge by facilitating the collection of relevant data without the expense of sending trained staff to conduct the observations required for intake. In the current study, non-technical researchers who were trained to deploy the system were able to do so for all the participant families without a problem. This indicates that support staff could be sent to homes to deploy the system rather than relying on expensive clinical staff either do the direct observation or deploy the select archiving system. A question that remains is whether the system can be installed and deployed by lay people without any specific training (for example, parents who receive the system in the mail). Another factor to be considered is that although these researchers who deployed the system did not have technical degrees they may be more technically savvy or motivated to learn how to deploy the system than the average person. One consideration is that "out of the box" selective archiving systems could be less effective in cases where a child's parents have limited technical know-how. 


\section{What Lies Ahead?}

This exploratory study shows there are two main contributions of this paper, one relating to the specific application domain of behavioral health, and one relating to the adoption of Ubicomp technologies in the real world. In this section we reflect on how our findings suggest the next steps for research in these two areas.

\section{Applying Capture and Access Technology to the Domain of Behavioral Health}

In this study we set out to determine what behavior parents found problematic and how this compared to a coding scheme used by behavior analysts. The potential advantages for selective archiving are clear. Families on the waiting list can use the system to collect initial data on their child's problem behaviors to facilitate the intake process, and families can avoid traveling to the behavior clinic for observation, where the behavior may not be observed. The clinic does not have to send a behavior analyst (an expensive and rare resource) to observe the behavior at the home, where their presence may impact the child's behavior and where the pressure to create behavioral samples are so strong that the parents are asked to recreate a situation in which the child is likely to exhibit the problem behaviors of concern. Our results show that without training, and over a period of just one day, most parents were able to flag at least one sample of each problem behavior of their child.

\section{Facilitating and Improving Capture of Severe Behavior Episodes}

Based on analysis of videos recorded at home using CRAFT and our discussion with behavior analysts, we draw several conclusions regarding the reasons behind the discrepancies between parents and behavior analysts' identification of problem behaviors. We believe that these examples reveal ways in which technology and research practices can be improved to better serve this population of users.

\section{Increased Deployment Length}

A thorough analysis of problem behavior by the severe behavior clinic requires more examples of the behavior than we typically saw in this study. That simply means that in practice, the selective archiving in the home would have to extend for multiple days, perhaps even weeks. Instances where parents flagged a behavior as problematic but behavior analysts did not agree are less critical in practice, a behavior analyst can easily dismiss them as unnecessary data collected by the 
parent. Instances where the parents failed to flag behavior that the analyst deemed as problem behavior, on the other hand, may be of concern because they indicate missed opportunities to record and share relevant samples. There are two ways we could improve the flagging of problem behavior by parents, better training or improved technology.

\section{To Train or Not To Train}

In previous research using selective archiving, the users and researchers agreed a-priori on what behaviors would be annotated [27]. In the current study, we opted to have parents use their intuitive sense of what constitutes "problem behavior." We did this because we wanted to describe the behaviors that parents considered to be problem behaviors and would cause them sufficient concern to bring their child to a severe behavior treatment clinic in the first place. By comparing the parent-annotated data against the behavior analysts' coding, we found numerous instances of mismatches. This raises the question of whether parent training may have been beneficial.

Our study shows that there were a number of reasons why discrepancies between a parent's annotation of problem behavior and the behavior analysts' assessment can occur. Parents may well recognize a problem behavior as such, but may be too busy dealing with the behavior in the moment to click the remote. For instance, in one video the mother was washing dishes while the father was busy talking on the phone. The child started shouting, which in two other instances the parent had flagged. However, this time, likely due to the distractions from other tasks, the parents verbally warned the child but did not flag the shouting. In another instance, a child was trying to undress at an inappropriate time (which was flagged as a problem behavior by the behavior analyst). The parent failed to flag this behavior although she may have recognized that it was problematic since she tried to stop the child from undressing.

While there may be multiple reasons for the mismatch between parents and behavior analysts, the examples above show that it is not clear that parent training would have solved all discrepancies between parents and behavior analysts. Training can be used to help parents understand what the clinical definition of a given severe behavior is and under what circumstances a behavior does not meet criteria. However, it is not clear that training would alleviate mismatches that are a result of overwhelmed or busy parents. Moreover, even when parents and analysts do not agree on what constitutes a problem behavior, it may be important for the analyst to learn what behaviors parents are struggling with and what they term misbehavior. 
Researchers must also bear in mind that training bears an additional cost. In our study, the trained behavior analyst went to the family's home to install the system and show parents how to use it. In practice, less costly support staff could be sent to the homes to deploy the system or the system could be shipped directly to the parents with instructions for how to install and operate it. Also, as mentioned earlier, longer deployments may lead to more Matches, which in turn could compensate for Mismatches.

If studies of longer deployment indicate that training is indeed necessary to improve parentcollected video data, then our study suggests a number of training experiences that seem likely to improve data collection. These include providing parents with clear definitions and sample videos of the target behaviors, as well as training videos that clarify how the system should be operated, including the cameras' field of view, to underscore the importance of selecting appropriate locations in the home for maximum coverage. In the future we plan to conduct both studies of extended deployments and of the impact of various parent training methods. The latter will include inexpensive methods of training such as printed material (using the definitions from Table 5) and multimedia versions that show still pictures and video examples of problem behavior.

\section{Automating Recognition of Behavioral Episodes}

A second approach to improving the capture of problem behaviors is to automate the recognition of the behaviors. The standard operational definitions of problem behaviors (Table 2) can give us $a$ priori information as to what forms of problem behaviors can be automatically detected (e.g. shouting can be detected using audio analysis). Our own observation of the video recording reveals that there might be detectable samples, in the form of gross movement (e.g., repeated banging of the head), exaggerated noises or parental utterances (e.g., "Don't bite mommy!") that signal behavior episodes of interest. Given that parents do identify some episodes correctly, we can adopt a supervised learning approach to help identify situations that appear similar. There are also opportunities to use sensors on the body of the child to help identify certain behavior patterns associated with physical movement, as demonstrated by Albinali et al. [33]. However, care should be taken to design on-body sensors that are safe for the child and that the child will tolerate wearing. In a previous study, we examined whether two particular types of on-body sensors, namely wrist-mounted accelerometers (to detect atypical hand movements) and a chest-mounted heart rate monitor (to detect deviations from the baseline heart rate) caused any reactivity from the child when worn [34]. The children in our study grew accustomed to the on-body sensors after a 
short period of time and showed no signs of discomfort. Additionally, parents, teachers and therapists showed high interest in using the on-body sensors as implicit triggers in a selective archiving system to collect behavior data.

Automated activity recognition could also prove to be an invaluable tool in addressing two categories of behavior that were particularly troublesome: Out of Scene and Alone. In reviewing the raw data of Table 5, there are two other cases that present opportunities for improving data collection. In situations in which a parent clicked the remote but the child was not visible in any camera view (Out of Scene), there is a question of whether the click was accidental or intentional. If intentional, then the camera placement was not ideal to capture the behavior. Recall that camera placement was determined by the parent, to allow them control over what they would feel comfortable having recorded. Feedback from the selective archiving could inform a parent that no child behavior was captured, assuming we could reliably determine that the child was not within camera field of view. Then the parents could decide to move the cameras to catch the behavior in the future.

Table 5 also shows instances in which a problem behavior was identified by the behavior analyst, but the parent was not in the view of the camera when the child was exhibiting problem behaviors (Alone). This was a prevalent occurrence for family F4, where no behaviors were identified by the parent, but seven episodes were identified by the analyst from the continuous recording but the parent was not around to witness it. The suggestions noted above for automated means of triggering recordings might well address such circumstances.

In conclusion, it is fitting to end this section by noting that the use of automated recognition to improve capture technology may be considered futuristic and implausible in real world settings (such as the home). In fact, such a real world deployment might be challenging given the limitations of this immature technology and other practical limitations such as the fact that using high quality sensors might be cost-prohibitive. However, we believe that this is one area where laboratory deployment will lead to important findings that can later be transferred and incorporated into real world deployments. In fact, our research group has started to investigate the role of automated recognition of social behavior in typically developing infants $[35,36]$. 


\section{Is Ubicomp Technology Ready for Real Use?}

We explicitly intended to design CRAFT for use by non-technical people. For Ubicomp technology to have its greatest impact, it needs to transition into the hands of people who can creatively use it. In our case, we wanted researchers from another discipline, behavioral psychology, to be comfortable with selective archiving and to have hands-on experience with a working research system to ask and answer research questions of interest to them. Our findings suggest that researchers were able to deploy CRAFT with minimal training and required no technical support during the deployments, and that parents were able to use the system to collect evidence of their child's problem behaviors. Results of this initial exploratory study show that the domain experts do understand the technology, are comfortable with it, and are able to define new and better hypothesis-driven studies for the future.

In designing CRAFT we have strived to provide seamless integration and use of technology. In our view, technology should allow parents to collect samples of problem behaviors in the course of their everyday activities and when their child is exhibiting problem behavior. Interestingly, we found that the video footage shed more light on how our system could be improved than parental or clinical report. For instance, in one case we saw a mother throwing the remote toward the father while she was busy restraining the child from engaging in the problem behavior. In another case the mother was busy cleaning when the child engaged in the problem behavior. Instead of stopping her activity to press the remote she threw it to the father so that he could click it. This suggests that during certain situations carrying and making a single click on the lightweight and compact remote can be inconvenient for the parent, and that we should consider allowing multiple individuals to make annotations.

\section{Conclusions}

We conducted an exploratory study to determine whether selective archiving, a form of automated capture and access that supports retroactive recording of significant events, was a feasible solution to enable parents to provide examples of problem behavior in the home to clinical professionals. While there are plenty of incentives to rely on parents to do this form of in-home data collection, and commercial products to support it, it is not known how well parents perform the data collection task. 
The results of this preliminary study, while not conclusive, do demonstrate the promise of selective archiving in the home. Our results show that without training, and over a period of just one day, most parents were able to flag at least one sample of each problem behavior of their child. Future work should focus on 1) developing systems that use timers or other sensing such that the duration of the deployment can be extended, 2) the development of precise experimental protocols that test the effectiveness of affordable training methods, 3) parent installation of selective archiving solutions and 4) computational techniques that complement a parent's ability to detect some relevant behavioral episodes to produce better recall of all relevant episodes. The automated techniques will likely require a combination of wearable and environmental sensing that determine relative location of people with respect to cameras in the environment as well as discovering pertinent behavioral patterns. While the latter may prove to be impractical in real world deployments, we consider that innovation as an important aspect of Ubicomp research.

Finally, we have also shown that careful attention to the design of a Ubicomp system for use by other domain specialists or non-technical users is key to moving Ubicomp research forward. We not only need to move our technologies from the lab to the real world, we need to move them out from under our protective control and into the hands and creative minds of other researchers and practitioners.

\section{Acknowledgements}

We would like to thank Gillian Hayes and Khai Troung for sharing their experiences on selective archiving and providing valuable suggestions. We also thank Yi Han for helping with the development of CRAFT.

Work reported in this manuscript was supported by an NSF Expeditions Award (1029679) and Children's Hospital of Atlanta Seed Grant Program.

\section{References}

1. Emerson E (1995) Challenging behaviour: Analysis and intervention in people with learning difficulties. New York, NY: Cambridge University Press.

2. McDougal J, Hiralall AS (1998) Bridging research into practice to intervene with young aggressive students in the public school setting: Evaluation of the behavior consultation team (BCT) Project. Annual Convention of the National Association of School Psychologists, Orlando, FL.

3. Horner RH, Carr EG, Strain PS, Todd AW, Reed HK (2002) Problem behavior interventions for young children with autism: A research synthesis. Journal of Autism and Developmental Disorders, 32(5), 423 - 446. 
4. Borthwick-Duffy SA (1996) Evaluation and quality of life: Special considerations for persons with mental retardation. In R. L. Schalock \& G. N. Siperstein (Eds.), Quality of life. Vol. I: Conceptualization and measurement (pp. 105-120). Washington, DC: American Association on Mental Retardation.

5. Koegel LK., Koegel RL, Surratt A (1992) Language intervention and disruptive behavior in preschool children with autism. Journal of Autism and Developmental Disorders, 22, 141153.

6. CDC, Prevalence of Autism Spectrum Disorder (2006) Autism and Developmental Disabilities Monitoring Network.

7. Sprague JR, Rian V (1993) Support systems for students with severe problem behaviors in Indiana: A descriptive analysis of school structure and student demographics. Indiana University Institute for the Study of Developmental Disabilities, Bloomington, IN.

8. Horner RH, Diemer SM, Brazeau, KC (1992) Educational support for students with severe problem behaviors in Oregon: A descriptive analysis from the 1987-88 school year. Journal of the Association for Persons with Severe Handicaps, 17, 154-169. Iwata, B. A., Dorsey,

9. Reichle J (1990) National Working Conference on Positive Approaches to the Management of Excess Behavior: Final report and recommendations. Minneapolis, MN: Institute on Community Integration, University of Minnesota.

10. Rojahn J (1994) Epidemiology and topographic taxonomy of self-injurious behavior. In T. Thompson \& D. Gray (Eds.), Destructive behavior in developmental disabilities: Diagnosis and treatment (pp. 49-67). Thousand Oaks, CA: Sage Publication.

11. Oliver C, Murphy GH, Corbett JA (1987) Self-injurious behaviour in people with mental handicap: A total population study. Journal of Mental Deficiency Research, 31, 147-162.

12. Sprague, JR, Horner RH (1999) Low-frequency high-intensity problem behavior: Toward an applied technology of functional assessment and intervention. In A. C. Repp \& R. H. Horner (Eds.), Functional analysis of problem behavior: From effective assessment to effective support (pp. 98-116).

13. Strain PS, Wolery M, Izeman S (1998). Considerations for administrators in the design of service options for young children with autism and their families. Young Exceptional Children, 1, 8-16.

14. Iwata BA, Worsdell AS (2005) Implications of Functional Analysis Methodology for the Design of Intervention Programs: Exceptionality Vol 13(1) 2005, 25-34.

15. Hanley GP, Iwata BA, McCord BE (2003) Functional analysis of problem behavior: A review: Journal of Applied Behavior Analysis Vol 36(2) Sum 2003, 147-185

16. Carey YA (2002) Effects of prior conditions on self-injurious behavior in subsequent analyses. Dissertation Abstracts International Section A: Humanities and Social Sciences.

17. Gresham FM, Watson TS, Skinner CH (2001) Functional Behavioral Assessment: Principles, procedures and Future directions. School of Psychology Review, 30, 156-172.

18. Iwata BA, Kahng SW, Wallace MD, Lindberg JS (2000) The functional analysis model of behavioral assessment. Reno, NV: Context Press

19. Patel MR, Carr JE, Kim C, Robles A, Eastridge D (2000) Functional analysis of aberrant behavior maintained by automatic reinforcement: Assessments of specific sensory reinforcers: Research in Developmental Disabilities Vol 21(5) Sep-Oct 2000, 393-407

20. Smith RG, Iwata B (1997) Antecedent influences on behavior disorders. Journal of Applied Behavior Analysis, 30, 343-375

21. Carr EG, Carlson JI, Langdon NA., Magito-McLaughlin D, Yarbrough SC (1998) Two perspectives on antecedent control: Molecular and molar. In J. K. Luiselli \& M. J. Cameron (Eds.), Antecedent control: Innovative approaches to behavioral support (pp. 3-28). Baltimore : Brookes

22. Hayes SC, Nelson RO, Jarrett RB (1987) The treatment utility of assessment functional approach to evaluating assessment quality, American Psychologist, 42, 963-974. 
23. Hintze JM (2005) Psychometrics of Direct Observation School Psychology Review, Volume 34 , No. 4, pp. 507-519

24. Foster SL, Cone JD (1986) Design and use of direct observation. In A.R. Ciminero, K.S. Calhoun, \& H. E. Adams (Eds), Handbook of behavioral assement (2nd ed., pp 253-354). New York: Wiley.

25. Kazdin AE (1982) observer effect: Reactivity of direct observation. New Directions for Methodology of social and Behavioral science, 14, 5-19.

26. Kazdin AE (1977) Artifact, bias, and complexity of assessment: The ABCs of reliability, journal of Applied Behavioral Analysis, 10, 141-150.

27. Hayes GR, Gardere L, Abowd GD, Truong KN (2008) CareLog: a selective archiving tool for behavior management in schools, in CHI '08, ACM: Florence, Italy. p. 685-694.

28. Hayes GR, Truong KN, Abowd GD, Pering T (2005) Experience Buffers: a Socially Appropriate, Selective Archiving Tool for Evidences-Based Care, in CHI '05 2005, ACM: Portland.

29. Hayes GR, Kientz JA, Truong KN, White DR, Abowd GD, Pering T (2004) Designing Capture Applications to Support the Education of Children with Autism, in Ubicomp '04, Springer Berlin / Heidelberg: Nottingham, England. p. 161-178.

30. Behavior Capture is a product of Behavior Imaging Solutions. (2011) https://www.behaviorimaging.com/html/index.htm. Accessed 20 February 2011

31. AviSynth. (2008), Available from: www.avisynth.org. Accessed 20 February 2011

32. Hayes GR, Abowd GD (2006) Tensions in Designing Capture Technologies for an EvidenceBased Care Community., CHI, ACM: Montreal, Canada.

33. Albinali F, Goodwin MS, Intille SS (2009) Recognizing stereotypical motor movements in the laboratory and classroom: a case study with children on the autism spectrum. 11th international conference on Ubiquitous computing. ACM: Orlando, Florida, USA. p. 71-80.

34. Nazneen, Boujarwah FA, Sadler S, Mogus A, Abowd GA, Arriaga RI (2010) Understanding the Challenges and Opportunities for Richer Descriptions of Stereotypical Behaviors of Children with ASD. 12th international ACM SIGACCESS conference on computers and Accessibility, ASSETS, Orlando, Florida, USA.

35. Wang P, Abowd GD, Rehg JM (2009) Quasi-Periodic Event Analysis for Social Game Retrieval. IEEE International Conference on Computer Vision, Kyoto, Japan.

36. Prabhakar K, Oh S, Wang P, Abowd GD, Rehg JM (2010) Temporal Causality for the Analysis of Visual Events. IEEE Conference on Computer Vision and Pattern Recognition (CVPR). 